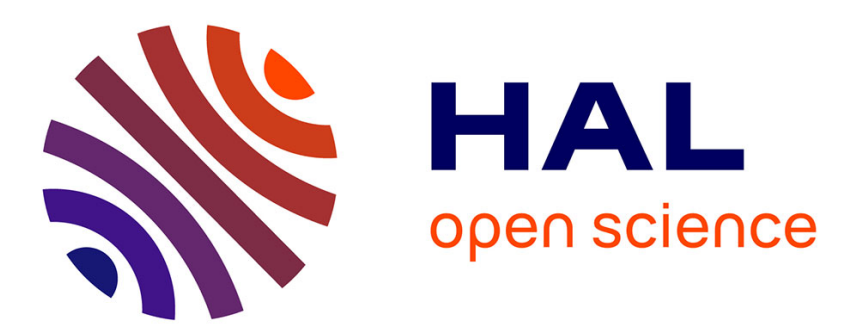

\title{
Electron density magnification of the collective spin-orbit field in quantum wells
}

Florent Baboux, Florent Perez, C. A. Ullrich, G. Karczewski, T. Wojtowicz

\section{To cite this version:}

Florent Baboux, Florent Perez, C. A. Ullrich, G. Karczewski, T. Wojtowicz. Electron density magnification of the collective spin-orbit field in quantum wells. Physical Review B: Condensed Matter and Materials Physics (1998-2015), 2015, 92 (12), pp.125307. 10.1103/PhysRevB.92.125307 . hal01324841

\section{HAL Id: hal-01324841 https://hal.sorbonne-universite.fr/hal-01324841}

Submitted on 3 Jun 2016

HAL is a multi-disciplinary open access archive for the deposit and dissemination of scientific research documents, whether they are published or not. The documents may come from teaching and research institutions in France or abroad, or from public or private research centers.
L'archive ouverte pluridisciplinaire HAL, est destinée au dépôt et à la diffusion de documents scientifiques de niveau recherche, publiés ou non, émanant des établissements d'enseignement et de recherche français ou étrangers, des laboratoires publics ou privés. 


\title{
Electron density magnification of the Coulomb-spin-orbit field in quantum wells
}

\author{
F. Baboux,${ }^{1, *}$ F. Perez,${ }^{1, \dagger}$ C. A. Ullrich, ${ }^{2}$ G. Karczewski, ${ }^{3}$ and T. Wojtowicz ${ }^{3}$ \\ ${ }^{1}$ Institut des Nanosciences de Paris, CNRS/Université Paris VI, Paris 75005, France \\ ${ }^{2}$ Department of Physics and Astronomy, University of Missouri, Columbia, Missouri 65211, USA \\ ${ }^{3}$ Institute of Physics, Polish Academy of Sciences, Warsaw, Poland
}

(Dated: May 31, 2016)

\begin{abstract}
The spin-orbit field acting on the spin waves of a spin-polarized electron gas is studied by inelastic light scattering on a CdMnTe quantum well. Above-barrier illumination allows us to vary the electronic density and control the collective Rashba and Dresselhaus coupling constants. We demonstrate that the enhancement between the single-particle and the collective spin-orbit field increases with increasing electronic density. This result is reproduced by a first-principles calculation. This behavior, which is opposite to usual Coulombic spin enhancements, reveals a novel aspect of the interplay of spin-orbit and Coulomb interactions in collective spin modes.
\end{abstract}

PACS numbers: 71.70.Ej 72.25.Rb 73.21.-b 78.30.-j

\section{INTRODUCTION}

Spin-orbit (SO) coupling is a relativistic effect: an electron moving with a velocity $\mathbf{v}$ in a static electric field $\mathbf{E}$ sees a magnetic field $\mathbf{B}_{\mathrm{SO}}=-\frac{1}{c^{2}} \mathbf{v} \times \mathbf{E}$ that couples to its spin ( $c$ is the speed of light). In zinc-blende type crystals such as GaAs or CdTe, intrinsic electric fields $\mathbf{E}$ arise from the lack of inversion center of the bulk material. ${ }^{1}$ In a heterostructure such as a quantum well, additional intrinsic electric fields result from the structural inversion asymmetry. ${ }^{2}$ The respective Dresselhaus and Rashba SO fields imprint the underlying heterostructure anisotropy onto the single- and many-particle properties of the carriers. ${ }^{3}$

At the single-particle level, the main effect of SO coupling is a momentum (k) dependent spin splitting and spin orientation of the conduction electron states, which can be described by an effective magnetic field $\mathbf{B}_{\mathrm{SO}}(\mathbf{k})$. Following early experimental evidence of this effect, ${ }^{4,5}$ recent experiments were able to separately measure the Rashba and Dresselhaus fields in the same sample, ${ }^{6,7}$ and the related anisotropy of the $g$-factor and of the spin relaxation rate. $^{8}$ In addition, these $\mathbf{k}$-dependent SO fields are known to be the major source of spin relaxation in doped quantum wells. Indeed, if a spin coherence is created, e.g. by aligning the individual spins of the electron gas, each spin will then precess with a proper direction and frequency, and the memory of the initial state will progressively be lost by decoherence. This single-particle effect, referred to as D'yakonov-Perel' decoherence, ${ }^{9}$ sets severe limitations on many applications in spintronics. ${ }^{10}$

Studies of SO effects at the many-body level - in the presence of Coulomb interactions between electrons are more preliminary, although they are expected to reveal a rich spectrum of phenomena. ${ }^{11}$ Coulomb interaction was shown to renormalize the magnetic quantities related to SO coupling, leading to enhancements of the SO coupling constants ${ }^{12}$ and of the $g$-factor. ${ }^{13}$ On the other hand, Coulomb interaction reduces D'yakonov-Perel' dissipation via the additional momentum scattering ${ }^{14,15}$ and the exchange field ${ }^{14,16}$ it produces. But the interplay of
Coulomb and SO interactions can even have more profound effects in the case of spin waves, where the behavior of electrons is intrinsically collective. In doped semiconductors, such spin waves consist of a collective oscillation or precession of the spin densities. ${ }^{17,18} \mathrm{SO}$ coupling was predicted to deeply modify the nature of these excitations, leading to chiral collective modes. ${ }^{19-21}$ Conversely, it was shown that Coulomb interaction can induce a striking organization of the SO fields acting on a spin wave. $^{22-25}$ Indeed, for the two main kinds of collective spin modes of quantum wells (intra- and inter-subband), Coulomb interaction transforms the D'yakonov-Perel' decoherence scenario into a constructive scenario, where the spin dynamics is governed by a collective SO field $\mathbf{B}_{\mathrm{SO}}^{\text {coll }}(\mathbf{q})$. The latter field is proportional to the excitation momentum $\mathbf{q}$, and it adds up to other magnetic actions such as real magnetic fields ${ }^{24}$ or exchange fields from magnetic impurities. ${ }^{25}$

This collective SO field $\mathbf{B}_{\mathrm{SO}}^{\text {coll }}(\mathbf{q})$ has the same orientation, but a much higher magnitude than the singleparticle SO field $\mathbf{B}_{\mathrm{SO}}(\mathbf{q})$ acting ${ }^{26-28}$ on individual electrons. Indeed, it was shown that $\mathbf{B}_{\mathrm{SO}}^{\text {coll }}(\mathbf{q})=\mathcal{C} \mathbf{B}_{\mathrm{SO}}(\mathbf{q})$, with $\mathcal{C}=5.2$ for the GaAs quantum well of Ref. 24 and $\mathcal{C}=6.5$ for the CdMnTe quantum well of Ref. 25 . The magnitude of this enhancement $\mathcal{C}$ is surprisingly large, as Coulombic enhancements of spin quantities are typically $^{18,29-31}$ of the order of the Coulomb coupling constant $r_{s}=1 / \sqrt{\pi n_{2 \mathrm{D}}} a_{\mathrm{B}}^{*}$ (with $n_{2 \mathrm{D}}$ the electronic density), which measures the average distance between electrons in units of the effective Bohr radius $a_{\mathrm{B}}^{*}$, and gives an estimate of the ratio between the interaction and kinetic energies in the ground state. Yet, $r_{s}$ was much lower than $\mathcal{C}$ in the probed samples $\left(r_{s}=1.2\right.$ and 1.9 , respectively). In addition, the measured $\mathcal{C}$ is significantly larger than the recently predicted ${ }^{32-34}$ enhancement of singleparticle SO coupling constants, which does not exceed a few tens of percent for the considered values of $r_{s}$. Together with a few other recent experimental studies, ${ }^{35,36}$ such strong enhancement suggests the existence of a cooperative mechanism between SO and Coulomb interactions, which is not accounted for theoretically yet. 
In this work, we gain insight into this novel mechanism, by providing experimental and theoretical evidence of an additional unconventional aspect: the enhancement $\mathcal{C}$ scales with the density $n_{2 \mathrm{D}}$ (i.e., with $1 / r_{s}$ ) rather than with $r_{s}$, in contrast with usual Coulombic spin enhancements. ${ }^{18,29-31}$ To demonstrate this behavior, we continuously tune the electron density of a CdMnTe quantum well through above-barrier illumination ${ }^{37,38}$ while monitoring the evolution of the collective SO field. The experimental findings are supported by a firstprinciples calculation of the enhancement factor, which is compatible with all previous theoretical and experimental results for the collective SO field. ${ }^{22-25}$ What is also important for applications, our results demonstrate the ability to efficiently control the collective SO field, with the electron density as the tuning parameter. This result extends to collective SO effects a result previously established only for single-particle SO fields ${ }^{39-41}$, and illustrates the potential of SO coupling to manipulate spin waves carrying logical information. ${ }^{42,43}$

\section{SAMPLE \& EXPERIMENTAL SETUP}

We investigate the two-dimensional electron gas (2DEG) embedded in a dilute magnetic quantum well of CdMnTe. ${ }^{25,31,44}$ In this system, the application of a moderate in-plane magnetic field (in the Tesla range) polarizes the spins localized on the Mn impurities, which in turn polarizes the electron gas through exchange interaction. ${ }^{45}$ This causes a giant Zeeman splitting $Z$ (of order $\mathrm{meV}$ ) of the electron gas, which dominates over the orbital quantization. One thus obtains a spin-polarized electron gas supporting spin-flip waves (SFW), which are collective precessions of the itinerant spins. ${ }^{25,31,44}$

Our sample is an asymmetrically modulation-doped, $20 \mathrm{~nm}$-thick $\mathrm{Cd}_{1-x} \mathrm{Mn}_{x} \mathrm{Te}(x \simeq 0.13 \%)$ quantum well grown along the [001] direction by molecular beam epitaxy. The sample is immersed in a superfluid helium bath $(\sim 2 \mathrm{~K})$. The electronic density is $n_{2 \mathrm{D}}^{0}=$ $2.7 \times 10^{11} \mathrm{~cm}^{-2}$ (as determined below, and in agreement with magneto-transport measurements) and the mobility is $1.7 \times 10^{5} \mathrm{~cm}^{2} / \mathrm{Vs}$.

We employ inelastic light scattering (ILS), which allows us to transfer a well-controlled momentum $\mathbf{q}$ to the spin excitations of the 2DEG. ${ }^{44,46}$ In our setup, depicted in Fig. 1(a), q can be varied both in magnitude and inplane orientation, measured by the angle $\varphi$ with respect to the [100] crystallographic direction of the well. A magnetic field $\mathbf{B}_{\text {ext }}$ is applied in the plane of the well, always perpendicular to q. The incoming and scattered light are cross-polarized, which matches the required selection rule to address spin-flip excitations. ${ }^{44,46}$
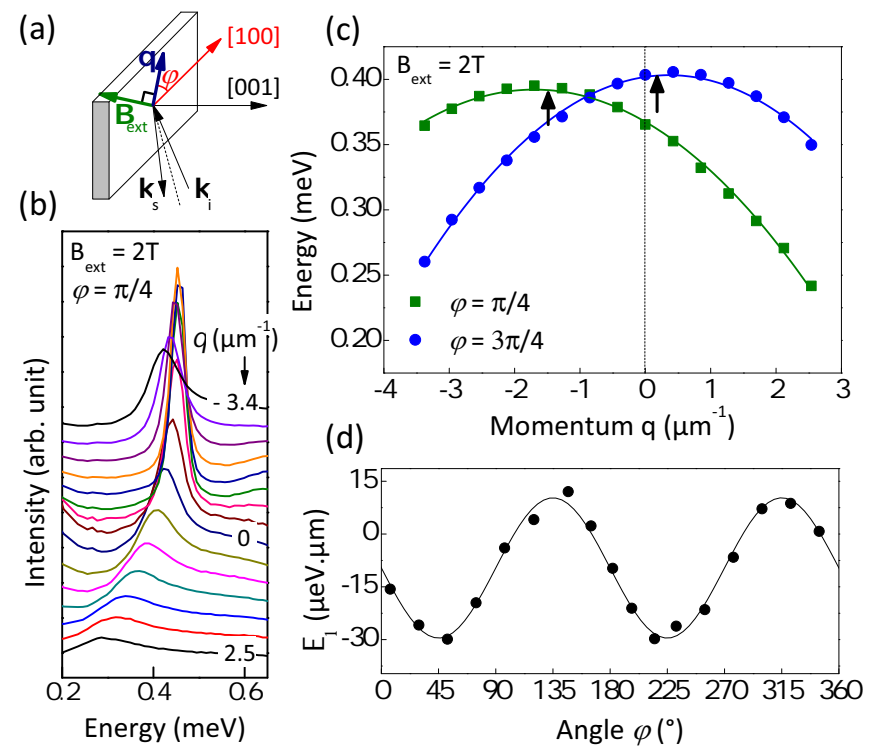

Figure 1. Determination of the collective spin-orbit field. (a) Inelastic light scattering (ILS) geometry: $\boldsymbol{k}_{i}$ and $\boldsymbol{k}_{s}$ are the incoming and scattered light wave-vectors, respectively; $\mathbf{q}$ is the transferred momentum, of in-plane orientation measured by the angle $\varphi$ from [100]. An external magnetic field $\mathbf{B}_{\text {ext }}$ is applied perpendicularly to $\mathbf{q}$. (b) ILS spectra of the spin-flip wave, obtained at $B_{\text {ext }}=2 \mathrm{~T}$ and $\varphi=\pi / 4$, for a series of transferred momenta $q$. (c) Wave-vector dispersion of the SFW for $\varphi=\pi / 4$ and $\varphi=3 \pi / 4$. (d) Variation of the linear term $E_{1}$ of the SFW dispersion (see text) as a function of the in-plane angle $\varphi$.

\section{EXPERIMENTAL RESULTS}

Figure 1(b) shows a series of ILS spectra of the SFW, obtained at fixed $B_{\text {ext }}=2 \mathrm{~T}$ and $\varphi=\pi / 4$, but for values of the transferred momentum varying between $q=-3.4 \mu \mathrm{m}^{-1}$ and $q=+2.5 \mu \mathrm{m}^{-1}$ [the positive sign for $q$ is defined by the orientation of $\mathbf{q}$ in Fig. 1(a)]. The corresponding wave-vector dispersion is plotted in Fig. 1(c) (squares): it is a parabola, with the maximum occurring at a non-zero value of $q$. In the same figure we show the dispersion measured for $\varphi=3 \pi / 4$ (circles): its maximum occurs for a different value of $q$. These characteristics are signatures of SO coupling. ${ }^{25}$ Indeed, in the absence of the latter, the dispersion $E(q)$ of the SFW would be independent of $\varphi$ and symmetric about $q=0$ : $E(q)=Z-f q^{2}$, where $Z$ is the Zeeman splitting of the conduction band ${ }^{45}$ and $f>0$ depends on $r_{s}$ and on the spin-polarization degree of the 2DEG. ${ }^{47}$

Due to SO coupling, the inversion asymmetry of the confining potential (Rashba effect) ${ }^{48}$ and of the crystalline cell (Dresselhaus effect) ${ }^{49}$ gives rise to an in-plane SO field acting on an electron of momentum $\mathbf{k}$ :

$$
\mathbf{B}_{\mathrm{SO}}(\mathbf{k})=2 \alpha\left(k_{y},-k_{x}\right)+2 \beta\left(k_{x},-k_{y}\right),
$$

(to lowest order in $\mathbf{k}$ ) with $\hat{x} \|[100]$ and $\hat{y} \|$ [010], and with $\alpha$ and $\beta$ the single-particle Rashba and Dresselhaus 
coupling constants, respectively. This field controls the spin splitting and orientation of individual electrons. ${ }^{3}$

By contrast, it was shown ${ }^{25}$ that the SFW, instead of feeling the distribution of the latter single-particle SO fields, is subject to a collective SO field

$$
\mathbf{B}_{\mathrm{SO}}^{\mathrm{coll}}(\mathbf{q})=2 \widetilde{\alpha}\left(q_{y},-q_{x}\right)+2 \widetilde{\beta}\left(q_{x},-q_{y}\right)
$$

where $\widetilde{\alpha}$ and $\widetilde{\beta}$ are the collective Rashba and Dresselhaus coupling constants, respectively. This collective SO field, which arises from many-body effects, adds up to the exchange field ${ }^{44,45}$ coming from the Mn impurities. This leads $^{25}$ to the emergence of a linear term in the SFW dispersion, such that:

$$
E(q, \varphi)=Z-f q^{2}-2(\widetilde{\alpha}+\widetilde{\beta} \sin 2 \varphi) q
$$

This linear term shifts the maximum of the SFW dispersion away from $q=0$, in an anisotropic way, see Fig. $1(\mathrm{c})$. We also note from the latter figure that the energy of the $q=0 \mathrm{SFW}$ mode slightly depends on $\varphi$, which is not accounted for by Eq. (3). This dependence, which evidences the breakdown of Larmor theorem ${ }^{44}$ due to SO coupling, will be detailed in a separate publication. ${ }^{50}$ Here we isolate the contribution of the collective SO field to the dispersion, by extracting the linear coefficient $E_{1}$ from a parabolic fit $E=E_{0}-f q^{2}+E_{1} q$. We repeat the procedure for a series of in-plane angles $\varphi$, and plot $E_{1}$ as a function of $\varphi$ in Fig. 1(d). The experimental variation $E_{1}(\varphi)$ is in remarkable agreement with the predicted sinusoidal variation $E_{1}(\varphi)=-2(\widetilde{\alpha}+\widetilde{\beta} \sin 2 \varphi)$. We deduce $\widetilde{\alpha}=46.7 \pm 1.2 \mathrm{meV} \AA$ and $\widetilde{\beta}=93.0 \pm 2.3 \mathrm{meV} \AA$, which completely characterizes the collective SO field of Eq. (2) acting on the SFW.

As a next step, we will vary the density of the $2 \mathrm{DEG}$ and determine how it affects this collective SO field. For this purpose we use a secondary cw green laser beam $(514.5 \mathrm{~nm})$, defocused to illuminate an area about 4 times larger than the one probed by ILS, guaranteeing the homogeneity of the 2DEG density on the probed area. ${ }^{37,38}$ To calibrate the effect of the above-barrier beam, we first show in Fig. 2(a) photoluminescence (PL) spectra taken for a series of green power densities $F_{\text {green }}$ ranging from 0 to $330 \mathrm{~mW} \mathrm{~cm}^{-2}$. The decrease of the quantum Stark effect blueshifts the PL peak, while the decrease of the 2DEG density narrows the PL line. Fitting the PL line shape $^{51}$ (black lines) yields the 2DEG density $n_{2 \mathrm{D}}$, shown in Fig. 2(c) with solid circles.

We confirm these determinations by extracting $n_{2 \mathrm{D}}$ from the cross-polarized ILS spectra of the single-particle excitations (SPE). Indeed, for a transferred momentum $q$, the corresponding SPE line peaks at $\hbar v_{\mathrm{F}} q$, with $v_{\mathrm{F}}$ the Fermi velocity. ${ }^{51}$ Figure 2(b) shows SPE spectra taken at $q=10.4 \mu \mathrm{m}^{-1}$ for various power densities $F_{\text {green }}$ [same color code as in Fig. 2(a)]. As expected, the energy of the peak decreases with increasing $F_{\text {green }}$. For each value of $F_{\text {green }}$, we extract the slope versus $q$ of the peak energy and correct it by taking into account Coulombic
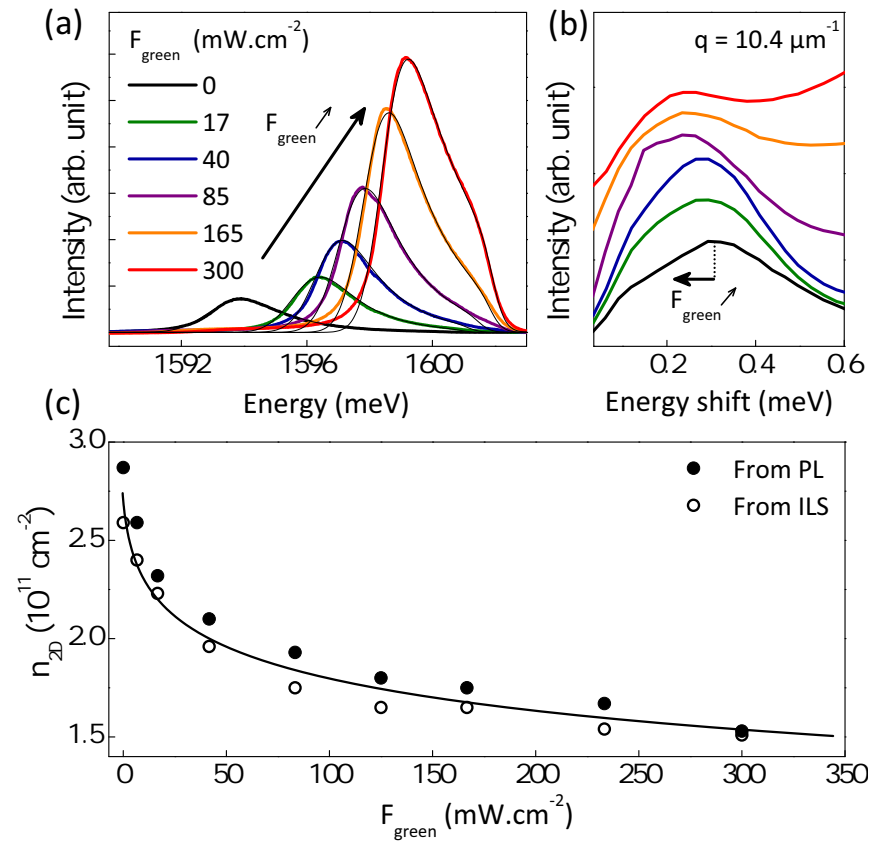

Figure 2. Tuning of the 2DEG density. (a) Photoluminescence (PL) spectra, at $B_{\text {ext }}=0$, for a series of power densities $F_{\text {green }}$ of secondary illumination. (b) For the same values of $F_{\text {green }}$, cross-polarized ILS spectra of the single-particle excitations at $B_{\text {ext }}=0$ and $q=10.4 \mu^{-1}$. (c) 2DEG density as a function of $F_{\text {green }}$, as extracted from the PL (solid circles) and ILS (empty circles) data. The line is a fit to the theory of Chaves et al. ${ }^{37}$

effects, ${ }^{47}$ to obtain $v_{\mathrm{F}}$. The corresponding values of $n_{2 \mathrm{D}}$ are plotted with empty circles in Fig. 2(c). Both determinations of the density are in good agreement, and we use their average in the following. Note that the measured variation of $n_{2 \mathrm{D}}$ with $F_{\text {green }}$ is well reproduced by the mechanism of Chaves et al. ${ }^{37}$ (see black line), which pre$\operatorname{dicts} F_{\text {green }}=A\left(n_{2 \mathrm{D}}^{0}-n_{2 \mathrm{D}}\right) e^{-B \sqrt{n_{2 \mathrm{D}}}}$, with $n_{2 \mathrm{D}}^{0}$ the $2 \mathrm{DEG}$ density in the absence of secondary illumination. Finally, fitting the energy $E_{0}$ of the $q=0 \mathrm{SFW}$ versus $B_{\text {ext }}$ with a Brillouin function, ${ }^{44,45}$ we verified that the green beam does not increase the temperature of the system by more than $0.3 \mathrm{~K}$. In conclusion, Fig. 2(c) demonstrates that $n_{2 \mathrm{D}}$ can be reproducibly tuned by a factor of 2 in our sample.

We are now in a position to determine how this change of $n_{2 \mathrm{D}}$ affects the collective SO field $\mathbf{B}_{\mathrm{SO}}^{\text {coll }}(\mathbf{q})$ acting on the SFW. For a series of values of $F_{\text {green }}$, we extract the collective coupling constants $\widetilde{\alpha}$ and $\widetilde{\beta}$ as described above, from the linear coefficient $E_{1}$ of the SFW dispersion at $B_{\text {ext }}=2 \mathrm{~T}$. Figure $3(\mathrm{a})$ shows the deduced $\widetilde{\alpha}$ (black squares) and $\widetilde{\beta}$ (red circles) as a function of $F_{\text {green }}$, and Fig. 3(b) shows the same quantities as a function of $n_{2 \mathrm{D}}$. Both $\widetilde{\alpha}$ and $\widetilde{\beta}$ exhibit a strong variation: the collective Dresselhaus constant $\widetilde{\beta}$ varies from 93 to $26 \mathrm{meV} \AA$, while the collective Rashba constant $\widetilde{\alpha}$ varies from $47 \mathrm{meV} \AA$ to nearly zero. 

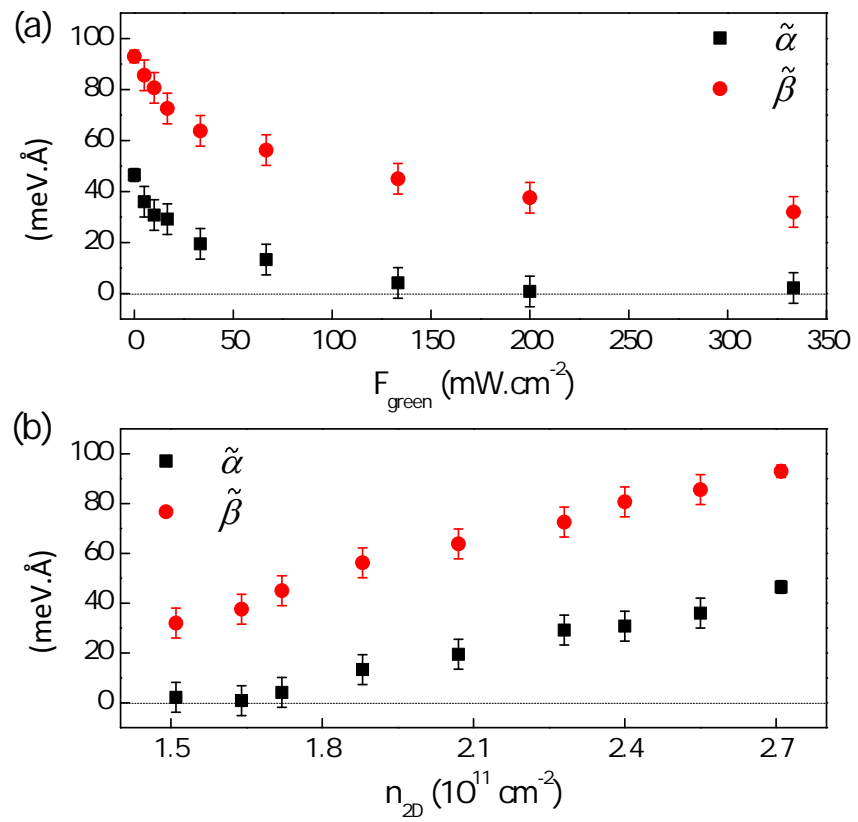

Figure 3. Optical control of the collective spin-orbit field. (a) Collective Rashba $(\widetilde{\alpha})$ and Dresselhaus $(\widetilde{\beta})$ coupling constants as a function of the secondary illumination power density. (b) Same quantities as a function of the 2DEG density, as deduced from Fig. 2(c).

Having determined the collective SO coupling constants for different values of the 2DEG density, we now turn to comparing them to the single-particle coupling constants $\alpha$ and $\beta$ [Eq. (1)], so as to deduce the enhancement factor $\mathcal{C} \simeq \widetilde{\alpha} / \alpha \simeq \widetilde{\beta} / \beta$ between the singleparticle and the collective SO field. ${ }^{24,25}$ In Ref. $25, \alpha$ and $\beta$ were determined experimentally, by analyzing the SO-induced splitting in the SPE line. This is not possible in the sample studied here, as the reduced disorder and stronger Coulomb interaction strength (higher $r_{s}$ ) lead to important modifications of the SPE line, departing its behavior from that of a Lindhard function. ${ }^{47}$ We will thus use theoretical values of the single-particle SO constants. For each value of $n_{2 \mathrm{D}}$, we perform a selfconsistent Schrödinger-Poisson calculation of the confining potential and electronic wavefunction. We then calculate the Rashba coefficient $\alpha=r_{41}^{6 c 6 c} e\left\langle E_{z}\right\rangle$ and the Dresselhaus coefficient $\beta=\gamma\left\langle k_{z}^{2}\right\rangle{ }^{3}$ Here, $r_{41}^{6 c 6 c}$ and $\gamma$ are material-dependent parameters, $e$ is the electronic charge and $E_{z}, k_{z}$ are respectively the electric field and wavevector along the growth axis. Using $r_{41}^{6 c 6 c}=6.93 \AA^{2}$ and $\gamma=43.9 \mathrm{eV \AA}^{3}$ calculated by $\mathbf{k} \cdot \mathbf{p}$ perturbation theory ${ }^{3}$ for CdTe, we show $\alpha$ and $\beta$ as a function of the electron density in the inset of Fig. 4.

We can now plot in Fig. 4 the enhancement factor $\mathcal{C}$ of the collective $\mathrm{SO}$ field, as given by $\widetilde{\alpha} / \alpha$ (black) and $\widetilde{\beta} / \beta$ (red). As the central result of this work, we observe a strong increase of the enhancement factor with the electronic density. As $\alpha$ and $\beta$ subtly depend on the exact shape of the wavefunction and confining potential, ${ }^{52}$ which are calculated here in a simple model, we cannot assert the relevance of the discrepancy between $\widetilde{\alpha} / \alpha$ and $\widetilde{\beta} / \beta$, nor their precise dependence with the density. But the increasing behavior with density is clearly evidenced, with $\mathcal{C}$ growing from $2-5$ to $10-20$ when $n_{2 \mathrm{D}}$ increases from $1.5 \times 10^{11} \mathrm{~cm}^{-2}\left(r_{s}=2.9\right)$ to $2.7 \times 10^{11} \mathrm{~cm}^{-2}\left(r_{s}=2.2\right)$. This behavior is quite remarkable, as it is opposite to usual Coulombic spin enhancements, which decrease when the electronic density is increased. ${ }^{18,29-34}$

Before continuing our discussion, we note that our modeling neglects the cubic Dresselhaus effect, as usually done in similar experiments. ${ }^{28,53}$ This effect would give an additional contribution $\gamma\left(-k_{x} k_{y}^{2}, k_{y} k_{x}^{2}\right)$ to the singleparticle SO field of Eq. (1). One could expect that this would translate into a cubic component in the collective SO field of Eq. (2) and thus in the dispersion of Eq. (3). However, it is experimentally seen that the dispersions are very well reproduced by simple parabolas (see Fig. 1c), so that it does not seem necessary to introduce a cubic term in the definition of the collective SO field. Regarding now the single-particle SO field, we note that if the cubic Dresselhaus effect is taken into account, an electron at the Fermi level acquires a SO splitting $2 \beta^{\star} k_{\mathrm{F}}$, with $\beta^{\star}=\gamma\left(\left\langle k_{z}^{2}\right\rangle-\pi n_{2 \mathrm{D}}\right)$. Here, $\beta^{\star}$ appears as a "renormalized" Dresselhaus coefficient, smaller than the linear coefficient $\beta$. In our experiments we calculate $\beta^{\star} \simeq 0.7 \beta$ for the lowest electronic density and $\beta^{\star} \simeq 0.6 \beta$ for the highest density explored. This means that, if the ratio $\widetilde{\beta} / \beta^{\star}$ had been considered (instead of $\widetilde{\beta} / \beta$ ), the values of the deduced enhancement factor $\mathcal{C}$ would be higher and show a steeper increase with the density, thus resembling more the enhancement deduced from the Rashba term $\widetilde{\alpha} / \alpha$ (see Fig. 4). Thus, the qualitative increase of the enhancement factor $\mathcal{C}$ with the density is fully preserved (even amplified) if cubic terms are considered.

\section{THEORETICAL DISCUSSION}

We note that a similar density dependence of SO effects on the SFW was found in Ref. 54, in analogous CdMnTe quantum wells. The authors studied the modulation of the SFW energy with the in-plane angle $\varphi$ (as in Ref. 25) at a fixed momentum $q=5 \mu \mathrm{m}^{-1}$, but as a function of $n_{2 \mathrm{D}}$ through above-barrier illumination. However, they attributed the amplitude $\Delta E$ of this modulation (energy difference of the SFW between $\varphi=\pi / 4$ and $\varphi=3 \pi / 4$ ) to the SO splitting of individual electrons at the Fermi momentum $\mathbf{k}_{\mathrm{F}} \| \mathbf{q}$. This single-particle interpretation yields $\Delta E=4 \beta k_{\mathrm{F}}=4 \beta \sqrt{2 \pi n_{2 \mathrm{D}}}$, which indeed reproduces the behavior of $\Delta E$ with $n_{2 \mathrm{D}}$. However, this picture disagrees with our data and model, which demonstrate the proportionality of $\Delta E$ to the momentum magnitude $q$ [Fig. 1(c)]. We note that Ref. 54 did not investigate the $q$-dependence of $\Delta E$. On the other hand, our model [Eq. (3)] predicts $\Delta E=4 \widetilde{\beta} q=4 \mathcal{C} \beta q$ and, as $\beta$ is mainly independent of $n_{2 \mathrm{D}}$ (inset of Fig. 4), the increase of $\Delta E$ 


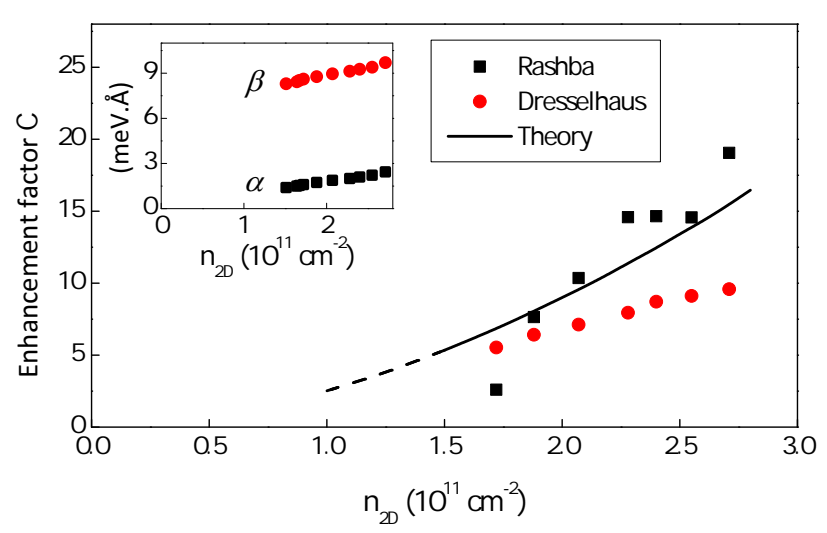

Figure 4. Enhancement factor $\mathcal{C}$ of the collective spinorbit field, obtained from $=\widetilde{\alpha} / \alpha$ (black squares) and $=\widetilde{\beta} / \beta$ (red circles) as a function of the 2DEG density $n_{2 \mathrm{D}}$. The black line corresponds to the theory of Eq. (4). Inset: calculated single-particle Rashba ( $\alpha$, black) and Dresselhaus $(\beta$, red) coefficients versus $n_{2 \mathrm{D}}$.

with increasing $n_{2 \mathrm{D}}$ demonstrates the increase of the enhancement factor $\mathcal{C}$ of the collective SO field acting on the SFW.

We now turn to the mechanism governing $\mathcal{C}$. It would be tempting to understand it as an effect of summation of the single-particle SO fields, but this interpretation is not physical, as SO fields only appear in the reference frame of each electron and cannot add up. However, in the presence of Coulomb interaction, each spin acts on the others through an exchange field, which tends to minimize the total Coulomb energy by aligning the spins parallel to each other. ${ }^{18}$ Due to SO coupling, $\mathbf{k}$ states participating to a collective mode experience the same change of their individual SO field $\mathbf{B}_{\mathrm{SO}}(\mathbf{k}+\mathbf{q})-\mathbf{B}_{\mathrm{SO}}(\mathbf{k}) \simeq \mathbf{B}_{\mathrm{SO}}(\mathbf{q}){ }^{22,24,25}$ Thus, the dynamical Coulomb exchange field aligns with the common $\mathbf{B}_{\mathrm{SO}}(\mathbf{q})$, and tends to average out the spread of orthogonal components. As a result, all the exchangecontributions will add up to create a Coulomb-SO field. We thus expect the enhancement factor $\mathcal{C}$ to scale with the exchange energy of the $2 \mathrm{DEG}$, i.e. to increase with the electronic density.

This heuristic argument, and the qualitative behavior of the enhancement $\mathcal{C}$, are fully corroborated by a first-principles calculation. We have extended the linearresponse formalism developed earlier for the case of intersubband excitations ${ }^{22,23,55}$ to calculate the spin-wave dispersions of a spin-polarized 2DEG in a quantum well with spin-orbit coupling. To first order in $\alpha$ and $\beta$ and to second order in the wavevector $\mathbf{q}$, we obtain after a somewhat involved calculation (to be published elsewhere) the following simple analytic result for the enhancement factor of the collective spin-orbit field:

$$
\mathcal{C}=\frac{2 E_{\mathrm{F}}}{Z^{*}} \frac{Z}{Z^{*}-Z}
$$

Here, $E_{\mathrm{F}}$ is the Fermi energy and $Z^{*}$ is the Coulombrenormalized Zeeman energy. The latter is given by $Z^{*}=Z+2 \partial \varepsilon_{\mathrm{xc}} / \partial \zeta$, with $\varepsilon_{\mathrm{xc}}$ the exchange-correlation energy per particle and $\zeta$ the spin-polarization degree of the electron gas $(-1 \leq \zeta \leq 1) .{ }^{18}$ As $Z$ is essentially independent of the density (within $3 \%$ ), we use its mean experimental value $Z=0.41 \mathrm{meV}$, and calculate $\mathcal{C}$ by using a quantum Monte-Carlo determination of $\varepsilon_{\mathrm{xc}}{ }^{29}$ The results are plotted with a black line in Fig. 4, in comparison with the quantities $\widetilde{\alpha} / \alpha$ and $\widetilde{\beta} / \beta$. The disagreement in the absolute values may arise from the uncertainty in the theoretical determination of $\alpha$ and $\beta$, as discussed above. On the other hand, the qualitative increase of $\mathcal{C}$ with the density is fully reproduced, confirming our interpretation. We note that our analytical finding [Eq. (4)] is fully compatible with the behavior found numerically in Ref. 22 for the magnitude of SO effects in the intersubband spin plasmons. This suggests the generality of the effect of Coulomb interaction on the collective spin excitations of conducting systems.

\section{CONCLUSION}

In conclusion, we have studied the SO fields acting on the spin excitations of a diluted magnetic quantum well under above-barrier illumination. The enhancement between the SO field acting on individual electrons and that acting on the collective spin mode has been determined for various electronic densities. Contrary to usual Coulombic enhancements of spin quantities, this enhancement increases with increasing density, as reproduced by a first-principles calculation. Together with the high values of the enhancement, this behavior evidences a novel aspect of the interplay of SO and Coulomb interactions in itinerant spin systems. An important consequence for spintronics applications is that we have evidenced, in usual semiconductors, SO coupling constants as high ${ }^{56}$ as $100 \mathrm{meV} \AA$, which can be tuned by varying the electron density. In the perspective of a spin-wave based transistor, ${ }^{42,43}$ our findings suggest the ability to switch such transistors by controlling either the density or the spin-orbit constants to tune the magnitude of the collective SO field.

\section{ACKNOWLEDGMENTS}

We thank Irene D'Amico for fruitful discussions and Mathieu Bernard \& Silbé Majrab for technical support. F.B. and F.P. acknowledge support from the Fondation CFM, C'NANO IDF and ANR. C.A.U. is supported by DOE Grant No. DE-FG02-05ER46213. The research in Poland was partially supported by the National Science Centre (Poland) through grant DEC2012/06/A/ST3/00247. 
* florent.baboux@lpn.cnrs.fr. Now at Laboratoire de Photonique et Nanostructures (CNRS/LPN), Marcoussis.

$\dagger$ florent.perez@insp.upmc.fr

1 G. Dresselhaus, Phys. Rev. 100, 580 (1955).

2 Y. Bychkov and E. I. Rashba, J. Phys. C 17, 6039 (1984).

3 R. Winkler, Spin-Orbit Coupling Effects in TwoDimensional Electron and Hole Systems (Springer, Berlin, 2003).

4 B. Das, D. C. Miller, S. Datta, R. Reifenberger, W. P. Hong, P. K. Bhattacharya, J. Singh, and M. Jaffe, Phys. Rev. B 39, 1411 (1989).

${ }^{5}$ P. D. Dresselhaus, C. M. A. Papavassiliou, R. G. Wheeler, and R. N. Sacks, Phys. Rev. Lett. 68, 106 (1992).

${ }^{6}$ L. Meier, G. Salis, I. Shorubalko, E. Gini, S. Schon, and K. Ensslin, Nat Phys 3, 650 (2007).

7 M. Studer, G. Salis, K. Ensslin, D. C. Driscoll, and A. C. Gossard, Phys. Rev. Lett. 103, 027201 (2009).

8 P. S. Eldridge, J. Hbner, S. Oertel, R. T. Harley, M. Henini, and M. Oestreich, Phys. Rev. B 83, 041301(R) (2011).

9 M. Dyakonov and V. Perel, Sov. Phys. Solid State 13 (1972).

10 I. Z̆utić, J. Fabian, and S. Das Sarma, Rev. Mod. Phys. 76, 323 (2004).

11 A. Agarwal, S. Chesi, T. Jungwirth, J. Sinova, G. Vignale, and M. Polini, Phys. Rev. B 83, 115135 (2011).

12 G. Liu, V. N. Antonov, O. Jepsen, and O. K. Andersen., Phys. Rev. Lett. 101, 026408 (2008).

13 B. Nedniyom, R. J. Nicholas, M. T. Emeny, L. Buckle, A. M. Gilbertson, P. D. Buckle, and T. Ashley, Phys. Rev. B 80, 125328 (2009).

14 M. Glazov and E. Ivchenko, Journal of Experimental and Theoretical Physics 99, 1279 (2004).

15 W. J. H. Leyland, G. H. John, R. T. Harley, M. M. Glazov, E. L. Ivchenko, D. A. Ritchie, I. Farrer, A. J. Shields, and M. Henini, Phys. Rev. B 75, 165309 (2007).

16 D. Stich, J. Zhou, T. Korn, R. Schulz, D. Schuh, W. Wegscheider, M. W. Wu, and C. Schller, Phys. Rev. B 76, 205301 (2007).

17 D. Stancil and A. Prabhakar, Spin Waves (Springer, New York, 2009).

18 G. Giuliani and G. Vignale, Quantum theory of the electron liquid (Cambridge University Press, Cambridge, 2005).

19 A. Ashrafi and D. L. Maslov, Phys. Rev. Lett. 109, 227201 (2012).

20 S. Maiti, V. Zyuzin, and D. L. Maslov, Phys. Rev. B 91, 035106 (2015).

21 S. Maiti and D. L. Maslov, Phys. Rev. Lett. 114, 156803 (2015).

22 C. A. Ullrich and M. E. Flatté, Phys. Rev. B 66, 205305 (2002).

23 C. A. Ullrich and M. E. Flatté, Phys. Rev. B 68, 235310 (2003).

24 F. Baboux, F. Perez, C. A. Ullrich, I. D'Amico, J. Gómez, and M. Bernard, Phys. Rev. Lett. 109, 166401 (2012).

${ }^{25}$ F. Baboux, F. Perez, C. A. Ullrich, I. D'Amico, G. Karczewski, and T. Wojtowicz, Phys. Rev. B 87, 121303(R) (2013).

26 V. Kalevich and V. Korenev, JETP Lett. 52, 230 (1990).

27 Y. K. Kato, R. C. Myers, A. C. Gossard, and D. D. Awschalom, Nature 427, 50 (2004).
${ }^{28}$ L. Meier, G. Salis, I. Shorubalko, E. Gini, S. Schön, and K. Ensslin, Nature Phys. 3, 650 (2007).

29 C. Attaccalite, S. Moroni, P. Gori-Giorgi, and G. B. Bachelet, Phys. Rev. Lett. 88, 256601 (2002).

30 J. Zhu, H. L. Stormer, L. N. Pfeiffer, K. W. Baldwin, and K. W. West, Phys. Rev. Lett. 90, 056805 (2003).

31 F. Perez, C. Aku-leh, D. Richards, B. Jusserand, L. C. Smith, D. Wolverson, and G. Karczewski, Phys. Rev. Lett. 99, 026403 (2007).

32 G.-H. Chen and M. E. Raikh, Phys. Rev. B 60, 4826 (1999).

33 C. H. Yang, W. Xu, Z. Zeng, and C. S. Tang, J. Phys.: Cond. Matt. 18, 6201 (2006).

34 A. Agarwal, S. Chesi, T. Jungwirth, J. Sinova, G. Vignale, and M. Polini, Phys. Rev. B 83, 115135 (2011).

35 B. Nedniyom, R. J. Nicholas, M. T. Emeny, L. Buckle, A. M. Gilbertson, P. D. Buckle, and T. Ashley, Phys. Rev. B 80, 125328 (2009).

${ }^{36}$ G. Liu, V. N. Antonov, O. Jepsen, and O. K. Andersen., Phys. Rev. Lett. 101, 026408 (2008).

37 A. Chaves, A. Penna, J. Worlock, G. Weimann, and W. Schlapp, Surf. Sci. 170, 618 (1986).

38 D. Richards, G. Fasol, and K. Ploog, Appl. Phys. Lett. 57, 1099 (1990).

39 J. Nitta, T. Akazaki, H. Takayanagi, and T. Enoki, Phys. Rev. Lett. 78, 1335 (1997).

40 M. Studer, G. Salis, K. Ensslin, D. C. Driscoll, and A. C. Gossard, Phys. Rev. Lett. 103, 027201 (2009).

41 A. Balocchi, Q. H. Duong, P. Renucci, B. L. Liu, C. Fontaine, T. Amand, D. Lagarde, and X. Marie, Phys. Rev. Lett. 107, 136604 (2011).

42 V. V. Kruglyak, S. O. Demokritov, and D. Grundler, J. Phys. D: Appl. Phys. 43, 264001 (2010).

43 Y. Kajiwara, K. Harii, S. Takahashi, J. Ohe, K. Uchida, M. Mizuguchi, H. Umezawa, H. Kawai, K. Ando, K. Takanashi, S. Maekawa, and E. Saitoh, Nature 464, $262(2010)$.

44 B. Jusserand, F. Perez, D. R. Richards, G. Karczewski, T. Wojtowicz, C. Testelin, D. Wolverson, and J. J. Davies, Phys. Rev. Lett. 91, 086802 (2003).

45 J. Gaj, R. Planel, and G. Fishman, Solid State Commun. 29, 435 (1979).

46 A. Pinczuk, S. Schmitt-Rink, G. Danan, J. P. Valladares, L. N. Pfeiffer, and K. W. West, Phys. Rev. Lett. 63, 1633 (1989).

47 F. Perez, Phys. Rev. B 79, 045306 (2009).

48 Y. Bychkov and E. I. Rashba, J. Phys. C 17, 6039 (1984).

49 G. Dresselhaus, Phys. Rev. 100, 580 (1955).

${ }^{50}$ It was shown in Ref. 25 that the modulation of the SFW energy is independent of $B_{\text {ext }}$ within the experimental error, which rules out any substantial influence of the g-factor inplane anisotropy (see for instance Ref. 53) neither in the zone-center energy nor in the linear term.

51 C. Aku-Leh, F. Perez, B. Jusserand, D. Richards, W. Pacuski, P. Kossacki, M. Menant, and G. Karczewski, Phys. Rev. B 76, 155416 (2007).

52 Our Schrödinger-Poisson calculation takes into account the nominal two Iodine modulation-doping layers (located at respectively 45 and $95 \mathrm{~nm}$ from the quantum well center), but ignores other possible unintentional charges, either negative at the surface of the sample [W. Maslana 
et al., Appl. Phys. Lett. 82, 1875 (2003)] or positive in the substrate. Also, as in similar experiments, ${ }^{28,53}$ we neglect a possible influence of the applied in-plane magnetic field, which can have orbital effects enhancing the Rashba coupling constant as shown e.g. in resonant tunneling diodes ${ }^{57,58}$.

53 P. S. Eldridge, J. Hbner, S. Oertel, R. T. Harley, M. Henini, and M. Oestreich, Phys. Rev. B 83, 041301(R) (2011).

54 C. Rice, D. Wolverson, A. Moskalenko, S. J. Bending, G. Karczewski, and T. Wojtowicz, Phys. Rev. B 87, 121304 (2013).
55 C. A. Ullrich, I. D'Amico, F. Baboux, and F. Perez, Proc. SPIE 8813, 88132W (2013).

56 In $\mathrm{Pt} / \mathrm{Co} / \mathrm{AlO}_{x}$ thin layers $\alpha$ is of order $400 \mathrm{meV} \AA$ [K.-W. Kim et al., Rev. Lett. 108, 217202 (2012)]; in $\mathrm{LaAlO}_{3} / \mathrm{SrTiO}_{3}$ hetrostructures $\alpha$ is about $20 \mathrm{meV} \AA$ [A. D. Caviglia et al., Phys. Rev. Lett. 104, 126803 (2010)].

57 J. Silvano de Sousa, H. Detz, P. Klang, E. Gornik, G. Strasser, and J. Smoliner, Applied Physics Letters 99, 152107 (2011).

58 M. A. Toloza Sandoval, A. Ferreira da Silva, E. A. de Andrada e Silva, and G. C. La Rocca, Phys. Rev. B 86, 195302 (2012). 